\title{
Cash is King - Auch im Controlling!
}

\section{Liebe Leserinnen und Leser,}

eine gute Zusammenarbeit zwischen Treasury und Controlling ist wichtiger denn je, wird aber selten thematisiert. Deshalb wollen wir uns im Schwerpunkt des vorliegenden Heftes just damit befassen und die Schnittstelle zwischen den beiden Funktionen näher betrachten. Um das Themenfeld von beiden Seiten aus angemessen zu beleuchten, sind die Auswahl der Schwerpunktbeiträge und das IM-DIALOG-Gespräch mit Rando Bruns von der Merck KGaA in enger Zusammenarbeit mit Professor Dirk Hachmeister von der Universität Hohenheim entstanden, der der Controlling \& Management Review auch als Beirat eng verbunden ist.

Anders als im Controlling ist im zentralen Treasury der Effizienzdruck in aller Regel vergleichsweise klein. $\mathrm{Zu}$ groß ist der finanzielle Hebel, den ein kleines Team stemmt. Dennoch sind die Veränderungen gerade in großen und global agierenden Unternehmen nicht zu vernachlässigen: Die Standardisierung globaler Prozesse, die hohe Volatilität der Finanzmärkte, die - höflich formuliert - unkonventionelle Politik der europäischen Zentralbank und nicht zuletzt immer neue Regulierungsvorhaben wie die European Market Infrastructure Regulation (EMIR) oder eine mögliche Finanztransaktionssteuer bringen enorme Herausforderungen und Chancen für das Corporate Treasury.

Gleichzeitig ist Cash als Steuerungsgröße mittlerweile auch im Controlling angekommen. Die zweite WHU-Zukunftsstudie (2014) zeigt, dass die Cash-Orientierung in der Unternehmenssteuerung von den befragten Controllern und CFOs heute fast durchgängig als eines der zentralen Zukunftsthemen des Controllings identifiziert wird. Vor einigen Jahren hatte sich dieses Bild noch deutlich differenzierter dargestellt. Cash-Orientierung ist dabei nicht nur das stärkere Augenmerk auf die eine oder andere Kennzahl. Vielmehr ist das Denken in Zahlungsströmen an der Universität und in der Unternehmenspraxis zunehmend an die Seite der traditionell dominierenden Brille des Rechnungswesens getreten, wozu

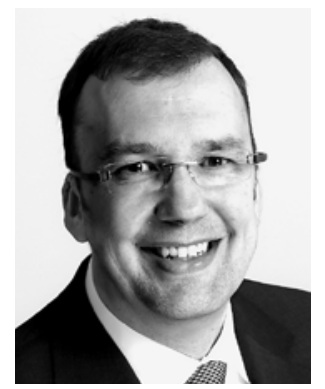

Utz Schäffer

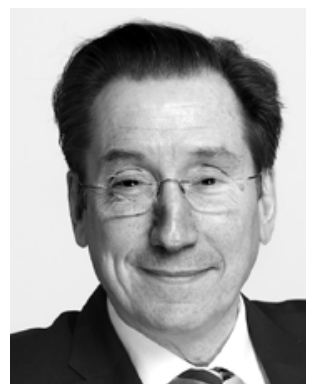

Jürgen Weber

nicht zuletzt die Finanzkrise, die zunehmende Bedeutung der Kapitalmärkte und die globale Verbreitung von angelsächsisch inspirierten Denkmustern beigetragen haben. Interessanterweise zeigt die WHU-Studie auch einen positiven $\mathrm{Zu}$ sammenhang zwischen der Nutzungsintensität von CashKennzahlen und dem Unternehmenserfolg: Cash ist King - nicht nur für das Treasury!

Warum also nicht eng zusammenarbeiten? Aus den verwandten Aufgabenbereichen von Treasury und Controlling ergeben sich sowohl mit Blick auf die relevanten Prozesse als auch mit Blick auf die organisatorische Zuordnung zahlreiche Schnittstellen, nicht zuletzt im Risiko-Management oder in der Liquiditätsplanung. Und auch für eine integrierte Personalentwicklung in der Finanzfunktion lohnt der Blick über den Tellerrand.

Viel Vergnügen bei der Lektüre wünschen Ihnen

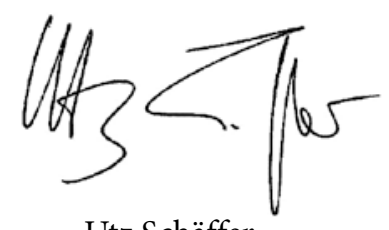

Utz Schäffer

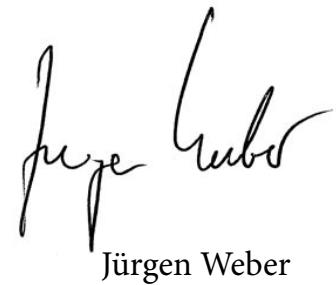

